\title{
Use of liposomal bupivacaine in the postoperative management of posterior spinal decompression
}

\author{
Anthony N. Grieff, BA, ${ }^{1}$ George M. Ghobrial, MD,1 and Jack Jallo, MD, PhD1,2 \\ ${ }^{1}$ Neurological Surgery, Thomas Jefferson University Hospital, and 'Department of Neurosurgery, Thomas Jefferson University, \\ Philadelphia, Pennsylvania
}

\begin{abstract}
OBJECTIVE The aim in this paper was to evaluate the efficacy of long-acting liposomal bupivacaine in comparison with bupivacaine hydrochloride for lowering postoperative analgesic usage in the management of posterior cervical and lumbar decompression and fusion.
\end{abstract}

METHODS A retrospective cohort-matched chart review of 531 consecutive cases over 17 months (October 2013 to February 2015) for posterior cervical and lumbar spinal surgery procedures performed by a single surgeon (J.J.) was performed. Inclusion criteria for the analysis were limited to those patients who received posterior approach decompression and fusion for cervical or lumbar spondylolisthesis and/or stenosis. Patients from October 1, 2013, through December 31, 2013, received periincisional injections of bupivacaine hydrochloride, whereas after January 1, 2014, liposomal bupivacaine was solely administered to all patients undergoing posterior approach cervical and lumbar spinal surgery through the duration of treatment. Patients were separated into 2 groups for further analysis: posterior cervical and posterior lumbar spinal surgery.

RESULTS One hundred sixteen patients were identified: 52 in the cervical cohort and 64 in the lumbar cohort. For both cervical and lumbar cases, patients who received bupivacaine hydrochloride required approximately twice the adjusted morphine milligram equivalent (MME) per day in comparison with the liposomal bupivacaine groups (5.7 vs $2.7 \mathrm{MME}, \mathrm{p}$ $=0.27$ [cervical] and 17.3 vs $7.1 \mathrm{MME}, p=0.30$ [lumbar]). The amounts of intravenous rescue analgesic requirements were greater for bupivacaine hydrochloride in comparison with liposomal bupivacaine in both the cervical (1.0 vs 0.39 MME, $p=0.31$ ) and lumbar (1.0 vs $0.37 \mathrm{MME}, p=0.08$ ) cohorts as well. None of these differences was found to be statistically significant. There were also no significant differences in lengths of stay, complication rates, or infection rates. A subgroup analysis of both cohorts of opiate-naive versus opiate-dependent patients found that those patients who were naive had no difference in opiate requirements. In chronic opiate users, there was a trend toward higher opiate requirements for the bupivacaine hydrochloride group than for the liposomal bupivacaine group; however, this trend did not achieve statistical significance.

CONCLUSIONS Liposomal bupivacaine did not appear to significantly decrease perioperative narcotic use or length of hospitalization, although there was a trend toward decreased narcotic use in comparison with bupivacaine hydrochloride. While the results of this study do not support the routine use of liposomal bupivacaine, there may be a benefit in the subgroup of patients who are chronic opiate users. Future prospective randomized controlled trials, ideally with doseresponse parameters, must be performed to fully explore the efficacy of liposomal bupivacaine, as the prior literature suggests that clinically relevant effects require a minimum tissue concentration.

http://thejns.org/doi/abs/10.3171/2015.11.SPINE15957

KEY WORDS liposomal bupivacaine; postoperative management; spondylolisthesis; Exparel; laminectomy; postoperative pain management; technique

$\mathrm{D}$ ECOMPRESSIVE laminectomy and fusion is a common procedure for the treatment of multilevel cervical and lumbar spine pathology. With posterior approaches there is considerable manipulation of the paraspinal muscles and disruption of the normal spinal anatomy, resulting in significant postoperative pain. The mechanism of postoperative pain is theorized to be a mul- tifactorial experience that results from noxious sensory signals from damaged tissue combined with modified central nervous system function. ${ }^{16}$

Lapses in acute postoperative pain control are encountered in up to $80 \%$ of patients with an additional $20 \%$ experiencing moderate pain. Several studies have shown that improved perioperative pain control is associated with a 
decreased risk of developing chronic pain, shorter hospital stays, faster recoveries, and both increased patient and physician satisfaction. Additional benefits to optimizing pain control may include improving cardiac, respiratory, and gastrointestinal function, as well as reducing the incidence of feared thromboembolic complications. ${ }^{11}$

In the past, high doses of single parenteral narcotics were the standard for pain management strategies. ${ }^{5}$ However, opioid medications carry significant adverse effects, including lethargy, constipation, pruritus, tolerance, withdrawal risk, and urinary retention. Recently, multimodal approaches, which function by using lower dosages of multiple agents with different mechanisms of action to modulate nociception, have gained popularity due to their favorable side effect profiles and superior analgesic properties. ${ }^{6}$ Within the multimodal approach to pain management in spinal surgery, the perioperative injection of local anesthetic agents has been shown to be both safe and effective. ${ }^{6,9,13}$

Liposomal bupivacaine (Exparel, Pacira Pharmaceuticals) has a nonopioid, lipid-based "honeycomb" structure that encapsulates bupivacaine and allows for delayed release and an effective duration of up to 96 hours. ${ }^{12,14}$ It was recently approved for local perioperative administration at the surgical site in concentrations up to $266 \mathrm{mg}$ for postoperative analgesia and has been most notably been studied for hemorrhoidectomy, bunionectomy, and total knee arthroplasty. $2,3,5,7,8$

In the era of cost-effective medicine, the potential of decreasing hospital length of stay and complications by promoting early mobilization after spinal surgery would be of great promise. As this agent has yet to be studied in any spine modalities, we sought to study the use of liposomal bupivacaine in posterior approach spinal surgery and evaluate its potential to decrease total narcotic pain medications use, achieve superior perioperative pain control, and decrease length of stay.

\section{Methods}

Institutional review board approval was obtained to conduct a retrospective, cohort-matched chart review of 531 consecutive cases over 17 months (October 2013 to February 2015) within an administrative database of spinal surgery procedures that were performed by a single surgeon (J.J.). Liposomal bupivacaine was administered to all patients undergoing posterior-approach cervical and lumbar spinal surgery at our institution beginning on January 1, 2014. Our protocol consisted of injecting $20 \mathrm{ml}$ liposomal bupivacaine (1.3\% concentration) into the paraspinal musculature at the operative levels prior to wound closure, rather than $20 \mathrm{ml}$ of $0.5 \%$ bupivacaine hydrochloride (Marcaine), which was used from October 2013 to January 1, 2014, thus creating 2 treatment arms designated by the use of liposomal bupivacaine or bupivacaine hydrochloride. The $20 \mathrm{ml}$ volume of liposomal bupivacaine was diluted in up to $40 \mathrm{ml}$ of normal saline to expand coverage for lumbar spinal surgeries greater than 2 levels. The injection sites included the subcutaneous tissues, epifascial and subfascial soft tissues, and paraspinal musculature.

As part of our multimodal pain protocol, all patients received preemptive patient-controlled anesthesia (PCA) and oral pain medications postoperatively as well. Patients who were receiving chronic narcotics preoperatively were maintained on the same regimen during their hospitalization.

All subjects were adults (age range 18-80 years) with an American Society of Anesthesiologists Physical Status Classification of I to III who were scheduled to undergo elective primary posterior decompression and fusion for spondylotic pathologies. All performed cervical surgeries were posterior cervical laminectomies with instrumented lateral mass screw-rod constructs with lateral mass fusion. Lumbar surgeries classified as a decompression alone consisted of either a bilateral laminoforaminotomy and medial facetectomy or a discectomy with either a laminectomy or hemilaminectomy. Surgeries described as a lumbar arthrodesis entailed transforaminal lumbar interbody fusion or instrumented posterolateral lumbar fusion. A JacksonPratt drain was left postoperatively in all fusion patients and removed at the discretion of the surgeon. Patients were further classified into those undergoing exposures within the anatomical levels of C3-T3 or L1-S3 to create the "cervical" and "lumbar" cohorts, respectively. Patients with previous surgical interventions at the same level or anterior approaches were excluded, as were patients with trauma/fractures/tumors and patients who did not receive a fusion. Those patients who experienced complications during hospitalization were also excluded from direct analysis of pain requirements and length of stay. Cohorts were further analyzed by preoperative opiate status.

Baseline demographics, as well as the outcome measurements of length of stay, days in the intensive care unit, discharge location, intravenous and oral opioid usage, and PCA days, were compared using the mean and standard deviation of each measurement by 2-tailed t-test analysis using Microsoft Excel. The mean postoperative analgesic consumption during hospitalization was calculated and normalized to $10 \mathrm{mg}$ of intravenous morphine (morphine milligram equivalent [MME]), as described in Table 1. For simplicity, all reported values were formatted in MME per day. Preoperative opiate usage was organized into 2 subgroups for analysis. Patients who used less than $10 \mathrm{MME}$ per day were considered opiate naive, while those using greater than or equal to $10 \mathrm{MME}$ per day were defined as opiate tolerant.

To account for variances in the size of surgical exposure and length of stay, the average opiate consumption was calculated on a milligram/day/level basis. Additionally, as our institution provides most patients with PCA by default, for which the actual milligrams of opiates received by the patient are not well documented, we accounted for this by subtracting the number of PCA days from the hospital length of stay. Two-tailed t-test analysis using Microsoft Excel (version 15.0.4737.1003) was performed.

\section{Results \\ Patient Characteristics}

One hundred sixteen patients were found to meet the inclusion criteria for this study. There were 52 patients in the cervical cohort and 64 patients in the lumbar cohort (Table 2). The respective mean ages of the bupivacaine hydrochloride and liposomal bupivacaine groups were 
TABLE 1. MME conversion table

\begin{tabular}{lc}
\hline \multicolumn{1}{c}{ Opioid \& Route } & Equivalency Factor to $10 \mathrm{mg}$ IV Morphine \\
\hline Morphine (IV) & $10 \mathrm{mg}$ \\
\hline Morphine (PO) & $30 \mathrm{mg}$ \\
\hline Hydromorphone (IV) & $1.5 \mathrm{mg}$ \\
\hline Hydromorphone (PO) & $7.5 \mathrm{mg}$ \\
\hline Oxycodone (PO) & $20 \mathrm{mg}$ \\
\hline OxyContin (PO) & $20 \mathrm{mg}$ \\
\hline Hydrocodone (PO) & $30 \mathrm{mg}$ \\
\hline Codeine (PO) & $200 \mathrm{mg}$ \\
\hline Tramadol (PO) & $500 \mathrm{mg}$ \\
\hline Fentanyl (IV) & $0.2 \mathrm{mg}$ \\
\hline Fentanyl (SC) & $0.4 \mathrm{mg}$ \\
\hline
\end{tabular}

IV = intravenous; $\mathrm{PO}=$ by mouth; $\mathrm{SC}=$ subcutaneous.

62.3 and 61.5 years for the cervical cohort and 63.1 and 61.9 years for the lumbar cohort. In the lumbar cohort, there was a significantly higher proportion of women than men $(p=0.021)$ in the group that received liposomal bupivacaine in comparison with bupivacaine hydrochloride. There were no other statistical differences found for any other parameters, including body mass index, average levels of spine exposure, chronic narcotics status, discharge location, insulin dependence, or smoking status (Table 2).

\section{Analgesic Requirements}

Neither PCA days nor levels of exposure were statistically different between groups. For both cervical and lumbar cases (Fig. 1), patients who received bupivacaine hydrochloride required approximately twice the adjusted MME per day in the bupivacaine hydrochloride arm in comparison with the liposomal bupivacaine arm (5.7 vs 2.7 MME, $\mathrm{p}=0.27$ [cervical] and 17.3 vs $7.1 \mathrm{MME}, \mathrm{p}$ $=0.30$ [lumbar]). The intravenous rescue pain medicine (Fig. 2) requirements (in addition to PCA) were increased in patients receiving bupivacaine hydrochloride in comparison with liposomal bupivacaine in the cervical (1.0 vs 0.39 MME, $p=0.31$ ) and lumbar (1.0 vs 0.37 MME, $p=$ 0.08 ) cohorts as well. However, none of these differences were found to be statistically significant (Table 3 ).

\section{Opiate-Dependent Versus Opiate-Naive Patients}

Patients who were preoperatively opiate naive or dependent were isolated for the subgroup analysis. In all cases, patients were maintained on their preoperative basal pain medication regimen, in addition to receiving increased intravenous and oral mediations; all narcotics administered were included in the analysis. For opiate-naive patients, the narcotic requirements were similar in the bupivacaine hydrochloride group in comparison with the liposomal bupivacaine group (Fig. 3) for both the cervical (2.3 vs 2.5 MME; $\mathrm{p}=0.87$ ) and lumbar cohorts (4.9 vs $3.12 \mathrm{MME}$; $\mathrm{p}=0.59$ ). In patients who were chronic preoperative opiate users, total opiate consumption in the bupivacaine hydrochloride group was less than the bupivacaine group for both the cervical (170.3 vs 3.2 MME; $p=0.24$ ) and lumbar (43.2 vs 8.8 MME; $p=0.26$ ) groups; however, the small sample size of this group and the differences in chronic narcotic requirements produced a large amount of variability and these values were not found to be statistically significant (Table 3).

\section{Length of Stay}

There was no significant difference in the lengths of stay between the bupivacaine hydrochloride and bupiva-

TABLE 2. Patient characteristics*

\begin{tabular}{|c|c|c|c|c|c|c|}
\hline \multirow[b]{2}{*}{ Variable } & \multicolumn{3}{|c|}{ Cervical Cohort } & \multicolumn{3}{|c|}{ Lumbar Cohort } \\
\hline & $\begin{array}{l}\text { Bupivacaine } \\
\text { Hydrochloride }\end{array}$ & $\begin{array}{l}\text { Liposomal } \\
\text { Bupivacaine }\end{array}$ & p Value & $\begin{array}{l}\text { Bupivacaine } \\
\text { Hydrochloride }\end{array}$ & $\begin{array}{c}\text { Liposomal } \\
\text { Bupivacaine }\end{array}$ & $\mathrm{p}$ Value \\
\hline No. of patients & 31 & 21 & & 34 & 30 & \\
\hline Male/female & $17: 14$ & $14: 7$ & 0.40 & $16: 18$ & $6: 24$ & $0.02^{*}$ \\
\hline Mean age (yrs) & 62.3 & 61.5 & 0.78 & 63.1 & 61.9 & 0.58 \\
\hline Mean body mass index $\left(\mathrm{kg} / \mathrm{m}^{2}\right)$ & 28.4 & 30.9 & 0.15 & 30.2 & 31.3 & 0.79 \\
\hline Mean levels exposed & 4.9 & 5.5 & 0.15 & 3.3 & 2.9 & 0.13 \\
\hline Active smoker & $2(6.5)$ & $4(19.0)$ & 0.20 & $3(8.8)$ & $3(10)$ & 0.80 \\
\hline Insulin dependent & $7(22.6)$ & $3(14.0)$ & 0.45 & $11(32.4)$ & $6(20)$ & 0.40 \\
\hline Chronic steroids & $3(9.7)$ & $2(9.5)$ & 0.97 & $2(5.9)$ & $0(0)$ & 0.16 \\
\hline Chronic narcotics & $7(22.6)$ & $7(33.3)$ & 0.41 & $11(32.4)$ & $8(25.8)$ & 0.75 \\
\hline Mean PCA (days) & 0.83 & 0.77 & 0.45 & 1.52 & 1.55 & 0.90 \\
\hline Hospital length of stay & 4.7 & 4.9 & 0.78 & 5.6 & 5.9 & 0.49 \\
\hline Discharge to home (rehabilitation) & $19(12)$ & $13(8)$ & 0.78 & $20(14)$ & $18(12)$ & 0.96 \\
\hline Infection rate & $5.9 \%$ & $4.3 \%$ & 0.80 & $7.9 \%$ & $8.8 \%$ & 0.55 \\
\hline Periop complications $†$ & $3(8.8)$ & $4(16.6)$ & 0.92 & $7(18.4)$ & $9(26.5)$ & 0.41 \\
\hline
\end{tabular}




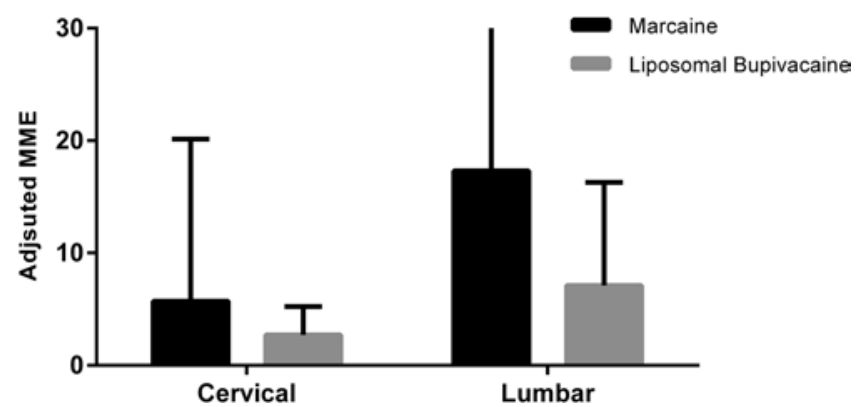

FIG. 1. Mean daily postoperative analgesia adjusted for PCA and levels exposed. The total daily pain medication requirements are shown. In the cervical cohort, patients treated with liposomal bupivacaine required $2.7 \pm 14.4$ MME per day in comparison with $2.706 \pm 2.5$ for bupivacaine hydrochloride (Marcaine) treatment; this is a $52.7 \%$ decrease. In the lumbar cohort, patients treated with liposomal bupivacaine required 7.1 $\pm 9.2 \mathrm{MME}$ per day compared with $17.3 \pm 55.3$ in patients who received bupivacaine hydrochloride; this is a $58.9 \%$ decrease.

caine groups in either the cervical (4.9 vs 4.7 days; $\mathrm{p}=$ 0.78 ) or lumbar (5.6 vs 5.9 days; $p=0.49$ ) cohorts (Fig. 4). The infection and complication rates between cohorts and treatment arms were also not significantly different between cohorts or treatments (Fig. 1). The most common complications encountered were dural tears, dysphagia, hypotension, postoperative fever, and postdischarge infection (Table 3).

\section{Discussion}

This retrospective, case-control study failed to demonstrate any significant differences between liposomal bupivacaine and standard bupivacaine hydrochloride in posterior-approach spinal surgery with regard to pain management at our institution. However, the trend toward decreased narcotic usage in the liposomal bupivacaine treatment arm warrants further investigation. Indeed, the total narcotic requirement was roughly double in the bupivacaine hydrochloride group in comparison with the liposomal bupivacaine group; these differences were striking, despite not reaching statistical significance. Due to this striking trend, further investigation is warranted with larger populations to see if this trend continues over a broad range of spinal procedures, which could account for the variability in narcotic use and lack of statistical significance. Moreover, we selected intravenous pain medication use as a proxy for assessing breakthrough pain and found that requirements were 3 times greater in the bupivacaine hydrochloride group than in the liposomal bupivacaine group as well, though these values also did not achieve statistical significance.

There was no decrease in the length of hospitalization between approaches, and the infection and complication rates were equivalent. However, in certain patient groups there may be an increased benefit for liposomal bupivacaine or bupivacaine hydrochloride at low levels. Specifically, in our study, we found that in both cohorts the preoperative opiate-dependent patients used significantly more narcotics prior to being treated with liposomal bupivacaine in comparison with bupivacaine hydrochloride, but narcotic use was equivalent between treatments in the opiate-naive group. While this was not a statistically sig-

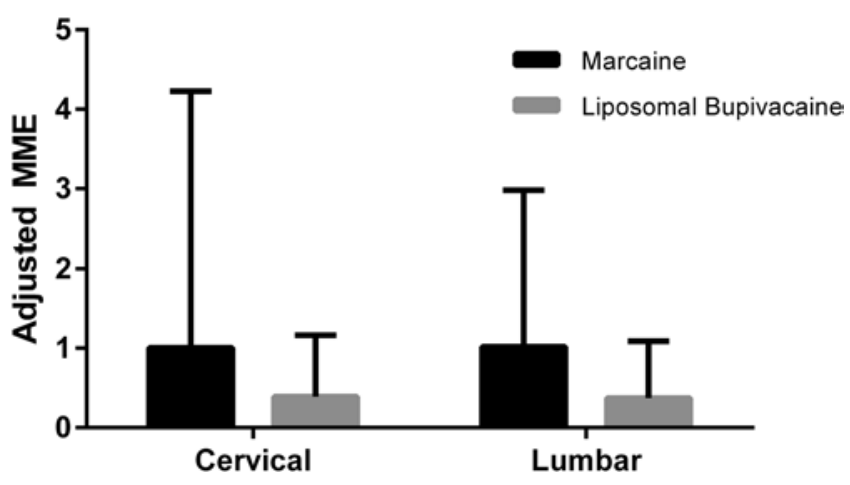

FIG. 2. Mean daily postoperative rescue pain medication adjusted for PCA and levels exposed. The intravenous rescue pain medication requirements are shown. In the cervical cohort, patients treated with liposomal bupivacaine required $0.40 \pm 0.78 \mathrm{MME}$ of intravenous rescue medication per day in comparison with $1.0 \pm 3.2$ for the bupivacaine hydrochloride treatment arm; this is an $81.1 \%$ decrease. The lumbar cohort patients treated with liposomal bupivacaine required $0.37 \pm 0.72$ MME of intravenous rescue pain medication per day in comparison with $1.0 \pm 1.96$ in patients who received bupivacaine hydrochloride; this is a $62.9 \%$ decrease.

nificant finding, the small sample size compared with the high degree of variability in narcotic use within patient cohorts may indicate a Type 2 error. Even if this is not statistically significant, this may be a clinically significant effect as these patients often require additional hospital services (e.g., acute pain service consultations, increased physical/occupational therapy usage) to manage their pain; these costs were not directly analyzed in this study. Therefore, the utility of liposomal bupivacaine in this special population requires further study, as it has the potential to tangibly decrease inpatient costs.

Recently, several other retrospective and prospective studies on total knee arthroplasty have made similar conclusions regarding the efficacies of liposomal bupivacaine and bupivacaine hydrochloride. ${ }^{1,15}$ In these studies, the authors hypothesized that the poor performance of liposomal bupivacaine may be accounted for by the decrease in the free drug concentrations at the injection site secondary to the slower release of bupivacaine from the liposomes, especially during the first 24 hours. ${ }^{10}$ However, the initiation lag of bupivacaine was not a relevant factor in our study because most patients received PCA for the first 24 hours postoperatively, regardless of the pain level.

The authors of a prospective study of total knee arthroplasty performed a dose-response analysis of liposomal bupivacaine and found that only $532 \mathrm{mg}$ liposomal bupivacaine-twice the concentration used in our protocol and that is currently approved by the FDA - was superior to standard bupivacaine on hospital Days 1 and $5 .^{3}$ In an analysis of 823 patients by Bramlett et al., doses up to 532 $\mathrm{mg}$ were found to be well tolerated with no increased incidence of either central nervous system or cardiac adverse events. ${ }^{3}$ It is possible that the administration of $266 \mathrm{mg}$ liposomal bupivacaine may fall below the threshold required to observe a clinically significant effect.

\section{Limitations}

We did not directly obtain patient-reported pain scores and relied upon rescue intravenous pain medication use as 
TABLE 3. Impact of liposomal bupivacaine, opiate-naive versus -tolerant status, and spinal region on postoperative narcotic use

\begin{tabular}{|c|c|c|c|c|c|c|}
\hline \multirow[b]{2}{*}{ Variable } & \multicolumn{3}{|c|}{ Cervical Cohort } & \multicolumn{3}{|c|}{ Lumbar Cohort } \\
\hline & $\begin{array}{l}\text { Bupivacaine } \\
\text { Hydrochloride }\end{array}$ & $\begin{array}{l}\text { Liposomal } \\
\text { Bupivacaine }\end{array}$ & $\begin{array}{c}p \\
\text { Value }\end{array}$ & $\begin{array}{l}\text { Bupivacaine } \\
\text { Hydrochloride }\end{array}$ & $\begin{array}{l}\text { Liposomal } \\
\text { Bupivacaine }\end{array}$ & $\begin{array}{c}p \\
\text { Value }\end{array}$ \\
\hline \multicolumn{7}{|l|}{ Opiate naive } \\
\hline No. of patients & 24 & 14 & 0.68 & 23 & 22 & 0.91 \\
\hline Preop narcotic use (MME/day) & 0 & 0 & & 0 & 0 & \\
\hline Postop narcotic use (MME/day) & 12.0 & 13.7 & 0.70 & 16.6 & 18.2 & 0.84 \\
\hline Postop narcotic use (MME/day/operative level) & 2.3 & 2.5 & 0.86 & 4.9 & 6.2 & 0.59 \\
\hline \multicolumn{7}{|l|}{ Opiate tolerant } \\
\hline No. of patients & 7 & 7 & 0.41 & 11 & 8 & 0.75 \\
\hline Preop narcotic use (MME/day) & 2.8 & 1.5 & 0.18 & 1.8 & 3.0 & 0.21 \\
\hline Postop narcotic use (MME/day) & 83.4 & 18.3 & 0.22 & 130.7 & 26.5 & 0.25 \\
\hline Postop (MME/day/operative level) & 17.3 & 3.2 & 0.24 & 43.2 & 8.8 & 0.26 \\
\hline
\end{tabular}

a proxy for lapses in pain control. These scores would be necessary to evaluate subjective patient satisfaction, which may provide justification for the increased cost of liposomal bupivacaine over bupivacaine hydrochloride.

The sample size was relatively small and the risk of a Type 2 error cannot be ruled out, especially because of the high variability in narcotic pain mediation use between chronic opioid users. While different mean analgesic requirements were noted, the average length of stay and complication rate were nearly identical, suggesting that even if liposomal bupivacaine is associated with decreased narcotic use that effect does not significantly decrease opioid-associated complications or decrease length of stay. Most patients postoperatively received PCA by default, but not all, which may have influenced the mean pain medication use in patients because the quantity dispensed was not readily available in our electronic medical records and patients certainly will have variable usage.

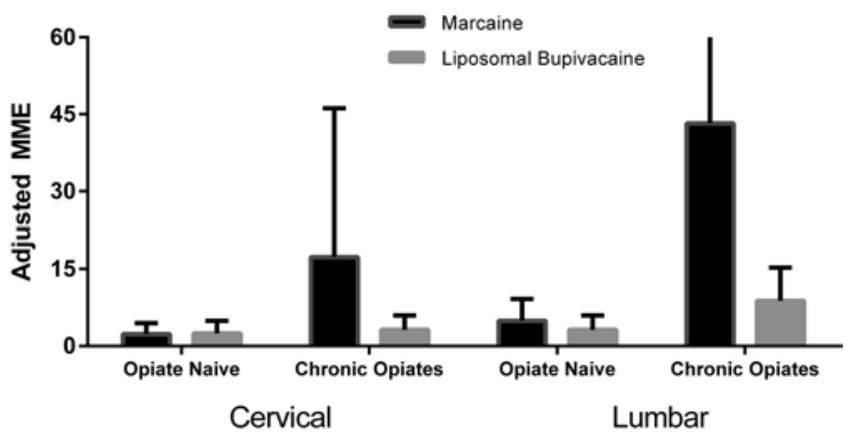

FIG. 3. Mean daily postoperative analgesia in opiate-naive and opiatedependent patients. Total pain requirements in patients receiving liposomal bupivacaine or bupivacaine hydrochloride relative to their preoperative opiate status are shown. In the cervical cohort, opiate-naive patients required $2.5 \pm 2.5 \mathrm{MME}$ for liposomal bupivacaine treatment in comparison with $2.4 \pm 2.1$ in the bupivacaine hydrochloride treatment arm. Chronic opiate users required 3.2 $\pm 2.8 \mathrm{MME}$ with liposomal bupivacaine in comparison with $17.3 \pm 28.9$ with bupivacaine hydrochloride treatment. In the lumbar cohort, opiate-naive patients required $3.2 \pm$ 10.3 MME with liposomal bupivacaine in comparison with $4.9 \pm 4.3$ MME with bupivacaine hydrochloride treatment. In chronic opiate users, those treated with liposomal bupivacaine required $8.8 \pm 6.46 \mathrm{MME}$ in comparison with $43.2 \pm 94.6 \mathrm{MME}$.
This study differs from other postoperative analgesia investigative studies, as we compared 2 drugs with equivalent mechanisms of action that vary only by pharmacokinetic action, whereas prior studies evaluated cohorts that received medications with dissimilar mechanisms of action. ${ }^{4}$

\section{Conclusions}

Liposomal bupivacaine did not significantly decrease perioperative opioid use, adverse events, or length of hospitalization within our multimodal pain management approach in patients who underwent cervical or lumbar laminectomy fusion. However, a trend toward decreased analgesic use with liposomal bupivacaine in comparison with bupivacaine hydrochloride was observed. Future investigations of patient and provider satisfaction would require further studies, as well as prospectively designed studies, in spinal populations to determine the significance of these trends.

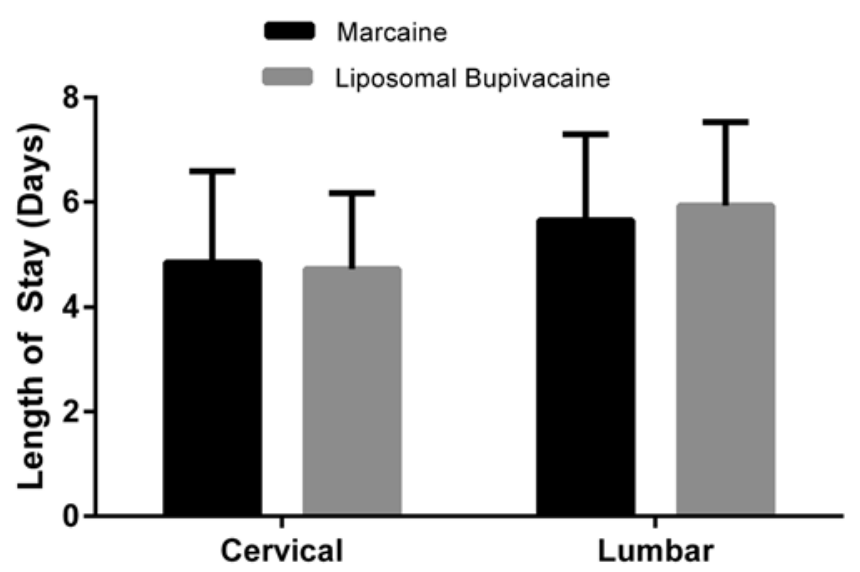

FIG. 4. Mean length of stay. To determine the average length of stay, the operative day was defined as Day 1. For the cervical cohort, the average length of stay was $4.7 \pm 1.5$ days for the liposomal bupivacaine treatment group and $4.8 \pm 1.75$ days for the bupivacaine hydrochloride treatment group. In the lumbar cohort, the average length of stay was $5.9 \pm 1.6$ days for the liposomal bupivacaine treatment group and $4.6 \pm$ 1.6 days for the bupivacaine hydrochloride treatment group. 


\section{References}

1. Bagsby DT, Ireland PH, Meneghini RM: Liposomal bupivacaine versus traditional periarticular injection for pain control after total knee arthroplasty. J Arthroplasty 29:16871690, 2014

2. Bergese SD, Ramamoorthy S, Patou G, Bramlett K, Gorfine SR, Candiotti KA: Efficacy profile of liposome bupivacaine, a novel formulation of bupivacaine for postsurgical analgesia. J Pain Res 5:107-116, 2012

3. Bramlett K, Onel E, Viscusi ER, Jones K: A randomized, double-blind, dose-ranging study comparing wound infiltration of DepoFoam bupivacaine, an extended-release liposomal bupivacaine, to bupivacaine $\mathrm{HCl}$ for postsurgical analgesia in total knee arthroplasty. Knee 19:530-536, 2012

4. Broome CB, Burnikel B: Novel strategies to improve early outcomes following total knee arthroplasty: a case control study of intra articular injection versus femoral nerve block. Int Orthop 38:2087-2089, 2014

5. Candiotti K: Liposomal bupivacaine: an innovative nonopioid local analgesic for the management of postsurgical pain. Pharmacotherapy 32 (9 Suppl):19S-26S, 2012

6. Devin CJ, McGirt MJ: Best evidence in multimodal pain management in spine surgery and means of assessing postoperative pain and functional outcomes. J Clin Neurosci 22:930-938, 2015

7. Golf M, Daniels SE, Onel E: A phase 3, randomized, placebo-controlled trial of DepoFoam ${ }^{\circledR}$ bupivacaine (extendedrelease bupivacaine local analgesic) in bunionectomy. Adv Ther 28:776-788, 2011

8. Gorfine SR, Onel E, Patou G, Krivokapic ZV: Bupivacaine extended-release liposome injection for prolonged postsurgical analgesia in patients undergoing hemorrhoidectomy: a multicenter, randomized, double-blind, placebo-controlled trial. Dis Colon Rectum 54:1552-1559, 2011

9. Lu S, Ma SC, Wang YY, Zhu ZH, Fan HW, Zhao GQ: Comparison of pain relief between patient-controlled epidural analgesia and patient-controlled intravenous analgesia for patients undergoing spinal fusion surgeries. Arch Orthop Trauma Surg 135:1247-1255, 2015

10. Mashimo T, Uchida I, Pak M, Shibata A, Nishimura S, Inagaki Y, et al: Prolongation of canine epidural anesthesia by liposome encapsulation of lidocaine. Anesth Analg 74:827834,1992
11. Ong CK, Lirk P, Seymour RA, Jenkins BJ: The efficacy of preemptive analgesia for acute postoperative pain management: a meta-analysis. Anesth Analg 100:757-773, table of contents, 2005

12. Portillo J, Kamar N, Melibary S, Quevedo E, Bergese S: Safety of liposome extended-release bupivacaine for postoperative pain control. Front Pharmacol 5:90, 2014

13. Ross PA, Smith BM, Tolo VT, Khemani RG: Continuous infusion of bupivacaine reduces postoperative morphine use in adolescent idiopathic scoliosis after posterior spine fusion. Spine (Phila Pa 1976) 36:1478-1483, 2011

14. Schmidt B, Ohri R, Wang JC, Blaskovich P, Kesselring A, Scarborough N, et al: Local pathology and systemic serum bupivacaine after subcutaneous delivery of slow-releasing bupivacaine microspheres. Anesth Analg 120:36-44, 2015

15. Schroer WC, Diesfeld PG, LeMarr AR, Morton DJ, Reedy ME: Does extended-release liposomal bupivacaine better control pain than bupivacaine after TKA? A prospective, randomized clinical trial. J Arthroplasty 30 (9 Suppl):64-67, 2015, 2015

16. Woolf CJ, Chong MS: Preemptive analgesia-treating postoperative pain by preventing the establishment of central sensitization. Anesth Analg 77:362-379, 1993

\section{Disclosures}

The authors report no conflict of interest concerning the materials or methods used in this study or the findings specified in this paper.

\section{Author Contributions}

Conception and design: Jallo. Acquisition of data: Grieff, Ghobrial. Analysis and interpretation of data: Grieff, Ghobrial. Drafting the article: Grieff, Ghobrial. Critically revising the article: all authors. Reviewed submitted version of manuscript: all authors. Study supervision: Jallo.

\section{Correspondence}

Jack Jallo, Department of Neurosurgery, Thomas Jefferson University Hospital, 909 Walnut St., 2nd Fl., Philadelphia, PA 19107. email: jack.jallo@jefferson.edu. 